\title{
Research governance impediments to clinical trials: a retrospective survey
}

\author{
Rustam Al-Shahi Salman ${ }^{1} \quad$ Timothy M Brock ${ }^{2} \quad$ Martin S Dennis ${ }^{3}$ \\ Peter A G Sandercock ${ }^{4}$ Philip M White ${ }^{5}$ Charles Warlow ${ }^{6}$
}

$J$ R Soc Med 2007; 100:101-104

\section{SUMMARY}

Objective We sought to evaluate the delays, between-centre variations in practice, and opportunity costs attributable to delays in research governance approval of clinical trials in the United Kingdom.

Design Retrospective survey.

Setting Research and Development (R\&D) departments at 50 UK National Health Service hospital trusts governing 57 hospital sites.

Participants R\&D departments participating in four randomized multicentre clinical trials coordinated by our Neurosciences Trials Unit.

Interventions None.

Main outcome measures Median delay between application and research governance approval.

Results Only half of the R\&D departments used the UK online R\&D form. Only a single copy of the application was required by $96 \%$ of R\&D departments. The median delay between application and research governance approval was 44 working days (interquartile range 23-80). A delay of $>20$ working days was incurred by 43 applications (75\%), of which 24 (56\%) were not explicable and 11 (20\%) were attributable to local funding negotiations.

Based on the trial randomization rates at each centre, 108 patients ( $17 \%$ of all patients randomized) could have been randomized during the delay, at a crude cost to funding agencies of $£ 53,743$; if a four week delay was deemed acceptable,

75 patients ( $12 \%$ of all patients randomized) could have been randomized during unacceptable delays, at a crude cost to funding agencies of $£ 37,700$.

Conclusions The UK research governance system incurs unacceptably long and costly delays for clinical trials. Urgent reform is needed, including rapid design and uniform implementation of the 'bureaucracy busting' measures in Best Research for Best Health.

${ }^{1}$ MRC Clinician Scientist, ${ }^{2}$ Medical Student, ${ }^{3}$ Professor of Stroke Medicine, ${ }^{4}$ Professor of Clinical Neurology, ${ }^{5}$ Consultant Neuroradiologist, ${ }^{6}$ Professor of Medical Neurology, Bramwell Dott Building, Division of Clinical Neurosciences, University of Edinburgh, Western General Hospital, Edinburgh EH4 2XU, UK

Correspondence to: Rustam Al-Shahi Salman

E-mail: Rustam.Al-Shahi@ed.ac.uk

\section{INTRODUCTION}

The future of academic medicine is under threat. ${ }^{1}$ There are concerns that both investigator-led as well as commercial research are relocating to parts of the world where research appears easier to do, to the detriment of UK health, wealth and research. ${ }^{2}$ Red tape has been one of the many recent impediments to furthering knowledge about the causes, prevention, and treatment of human illness in the UK. ${ }^{2}$ Slowing the conduct of clinical trials in particular runs the risk that the identification of beneficial treatments will be delayed, or the use of harmful treatments perpetuated.

The Department of Health has therefore promised 'bureaucracy busting' measures in its recent five-year vision of a future 'vibrant health research system' in Best Research for Best Health. ${ }^{3}$ A specific concern of this vision is the speed of the process of approval of clinical trials. There are misgivings about Best Research for Best Health because it seems to be targeted at commercially-led trials, ${ }^{2,4}$ overall expenditure on research promises to be lower, ${ }^{4}$ and the document only applies to England. ${ }^{2}$ Nevertheless, its vision of an expedited process of research governance in the UK would benefit all clinical trials.

The process of research ethics committee approval in the UK has improved. ${ }^{5}$ In the 1990 s, UK researchers encountered heterogeneous handling and judgement of their proposals, but now the Central Office for Research Ethics Committees (COREC) coordinates operational and infrastructure arrangements, implements standards to ensure national consistency, provides training for members of research ethics committees and identifies computerized solutions for procedural management.

Other impediments to clinical trials, however, are the processes of research governance approval, management approval and negotiation of service support costs. Without a COREC equivalent, research governance is currently decentralized, so accountability is unclear. ${ }^{6}$ Although many Research and Development (R\&D) departments are operating efficiently and effectively, ${ }^{7}$ others cause unexplained delays which could be unethical ${ }^{8}$ or even harmful. ${ }^{9}$ Some research governance, management and funding considerations inevitably rely on subjective judgements which will cause variation, ${ }^{10}$ and differences in organizational structure and management accountability between 
NHS trusts make internal authorization procedures heterogeneous (for example, in finance and pharmacy). But lengthy delays and poor process due to bureaucracy and inflexibility must be avoidable.

Given these concerns about research governance, and in view of the aspirations of Best Research for Best Health, we sought to evaluate the delays incurred in starting clinical trials in the era since COREC streamlined the process of research ethics committee appraisal with a new web-based form, in order to provide baseline data upon which the effects of Best Research for Best Health could be judged.

\section{METHODS}

In July-August 2006, we collected data from R\&D departments in the UK by telephone, post and email concerning their approval process for four multicentre randomized controlled clinical trials. The four trials coordinated by our Neurosciences Trials Unit are comparing general anaesthetic versus local anaesthetic for carotid surgery (GALA, www.galatrial.com); intravenous recombinant tissue plasminogen activator versus open control for acute ischaemic stroke (IST3, www.ist3.com); graduated compression stockings versus routine care for the prevention of deep vein thrombosis after stroke (CLOTS, www.clotstrial.com); and hydrocoils versus bare platinum coils in the endovascular treatment of intracranial aneurysms (HELPS, http://www.helpstudy.org/). We only collected data for UK centres that had applied for $\mathrm{R} \& \mathrm{D}$ approval after the introduction of the COREC system of ethical approval in 2000. Where exact dates were not recorded by $\mathrm{R} \& \mathrm{D}$ departments (if paper and/or electronic correspondence were unavailable to the officers at the R\&D departments at the time of this survey), mid-month dates were ascribed (7 of 57 dates of R\&D application [12\%] and 3 of 57 dates of R\&D approval [5\%]). We collected data on the randomization rate at each of these centres in each trial from the time randomization began until April 2006.

\section{RESULTS}

There were 57 hospitals participating in the four trials (20 in CLOTS, 15 in IST3, 12 in GALA and 10 in HELPS), relating to 50 individual NHS hospital trusts in England, Wales, Scotland and Northern Ireland. Of these 57 separate applications to R\&D departments, 37 (65\%) were made on a variety of paper forms designed by each $\mathrm{R} \& \mathrm{D}$ department, 14 (25\%) used the online R\&D application form and the remaining six $(10 \%)$ had simply required all or part of the COREC application form. Of the 18 applications made after March 2005 (when the online R\&D application form was introduced), nine R\&D departments (50\%) used the online system and the other half continued to use bespoke paper forms. Only two R\&D departments (4\%) required multiple copies of the application (five in one case and 13 in the other).

The median delay between application and research governance approval in all four trials was 44 working days (inter-quartile range [IQR] 23-80) (Figure 1), and there was a significant difference in this delay between the four trials (Kruskal-Wallis test, chi-squared $=10.4, \quad P=0.015)$ : GALA 25 days (IQR 10-35), IST3 44 days (IQR 15-55), HELPS 65 days (IQR 31-80) and CLOTS 68 days (IQR 37125). There was no correlation between the observed delay

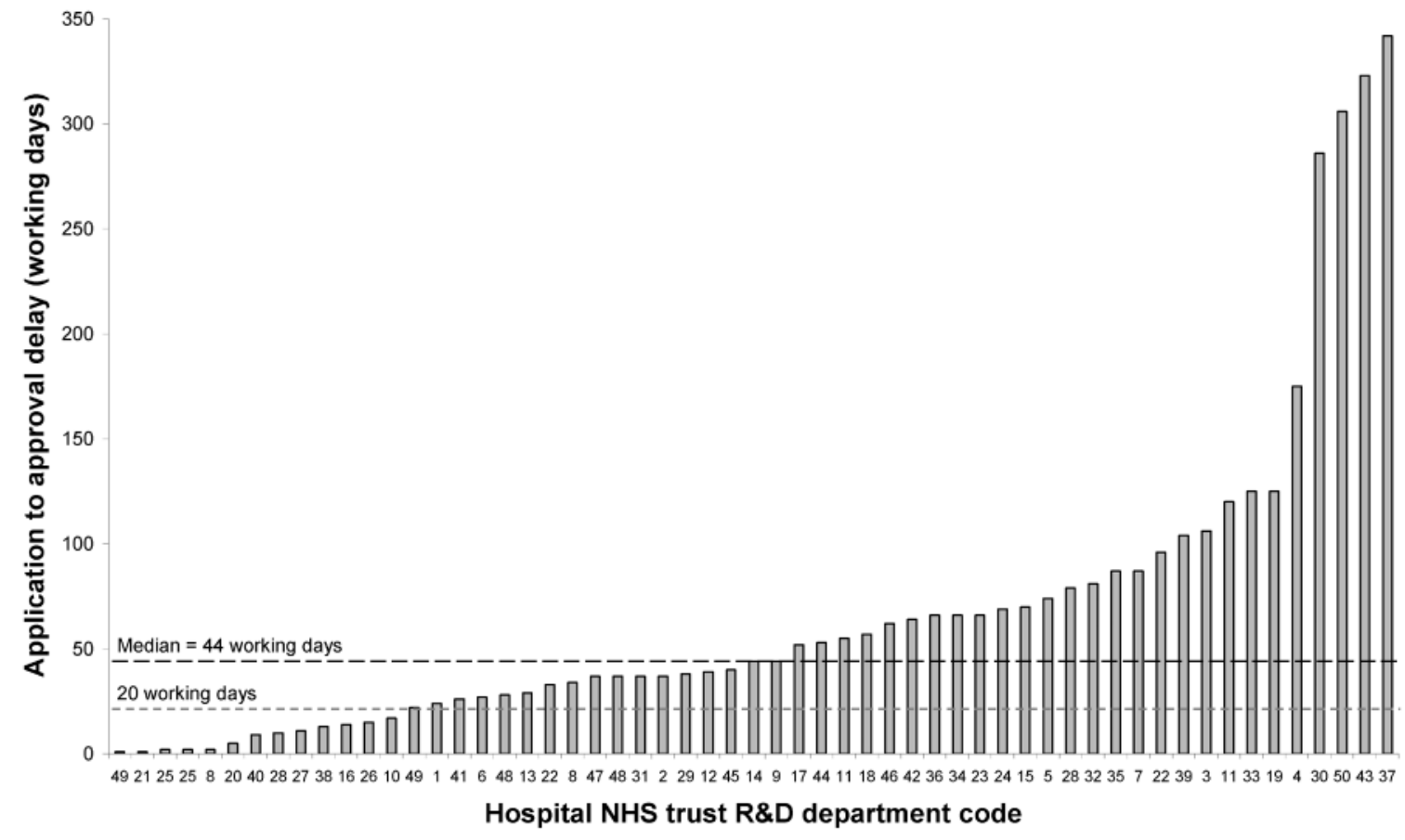

Figure 1 Number of working days taken to approve research governance applications for $\mathbf{5 7}$ hospitals within $\mathbf{5 0}$ NHS trusts 
and the date of application for research governance approval (Spearman's rank correlation coefficient $=-0.02, P=0.87$ ).

We investigated the reasons for any delays of over four weeks (20 working days) until approval was given, which involved 43 R\&D department applications (75\%) (Figure 1 ). More than one reason was given for the delays incurred at nine R\&D departments. Although $24 \mathrm{R} \& \mathrm{D}$ departments $(56 \%)$ could not give a reason for a delay of over four weeks, the most common reasons cited by the others were: local funding negotiations over support costs $(20 \%)$, R\&D department staff shortages/replacements (16\%), sitespecific ethical assessment of the trial was still awaited $(16 \%)$, the Principal Investigator made an error or omitted application materials $(14 \%)$, data protection checks were required (5\%), the $\mathrm{R} \& \mathrm{D}$ department was unclear about the trial's intervention (5\%), and an amendment was required (2\%). Four of the five longest delays (Figure 1) involved the CLOTS trial, and these delays were largely attributable to negotiations over service support costs.

In April 2006, randomization into the trials had not begun at eight centres, but at the other 49 centres the median duration of randomization was 14.3 months (IQR 9.5-21, range 1.5-67.5). Up to this time, 620 patients had been randomized at these centres, and the median number of patients randomized at each centre was nine (IQR 3-17). We multiplied the randomization rate at each individual hospital by the delay in obtaining approval from its own $\mathrm{R} \& \mathrm{D}$ department, in order to calculate the number of patients who could have been randomized during the whole time awaiting R\&D approval. The 'number not treated' (NNT) during the whole time spent waiting for R\&D approval was $108(17 \%$ [95\% CI 15-21\%] of all 620 patients randomized to date). If a four-week delay until R\&D approval is deemed acceptable, the NNT would have been $75(12 \%$ [95\% CI 10-15\%] of all 620 patients randomized to date).

We calculated a crude measure of the financial cost of these delays to funding agencies by multiplying the NNT in each trial by the approximate per capita cost in each trial (calculated by dividing the total funding for each trial by its target sample size). CLOTS cost $£ 276$ per patient $(£ 1,378,594 / 5,000)$, GALA cost $£ 286$ per patient $(£ 1,000,000 / 3,500)$, HELPS cost $£ 724$ per patient $(£ 362,000 / 500)$ and IST3 cost $£ 500$ per patient $(£ 2,998,073 / 6,000)$. The cost of the patients not randomized was $£ 53,743$ during the whole time awaiting $R \& D$ approval, or $£ 37,700$ if a four-week delay until $R \& D$ approval is deemed acceptable.

\section{DISCUSSION}

The processes of approval of clinical trials in the UK appear to vary significantly between R\&D departments and they are, in general, far too slow. We found that for every eight patients enrolled in the first few years of four clinical trials, one further patient could have been enrolled during unacceptable delays associated with the processes of research governance approval, management approval and negotiation of service support costs.

The Research Governance Framework for Health and Social Care was introduced in England in 2001 and later revised in 2005.11 This framework intended R\&D departments to take a 'verifying' role by managing and protecting high legal, ethical and scientific standards in research through monitoring and auditing, with each project's sponsor having a central role, as well as continuing to monitor research projects' resource use in the NHS. ${ }^{12}$ Interestingly, the R\&D Forum (an independent organization) and COREC have taken the initiative in trying to improve the process of research governance approval. COREC funded the creation of the online R\&D application form (https://www.rdform.org.uk/), which is populated by data from the online COREC form and asks standard questions about governance, management and funding at a local study site. However, the R\&D Forum does not have authority over NHS R\&D departments, so the use of the form is not mandatory.

Only half of the R\&D departments surveyed in our study appeared to employ efficient methods such as the online $\mathrm{R} \& \mathrm{D}$ application form. Variation in practice, unnecessary delays, and under-use of information technology are clear targets for the 'bureaucracy busting' developments promised by Best Research for Best Health. ${ }^{3}$

We are unaware of planned, formal evaluations of the UK research governance system, although other researchers have reported long delays, ${ }^{13-15}$ lost documentation, ${ }^{14,15}$ seemingly unnecessary honorary contracts and Criminal Records Bureau checks, ${ }^{13,16}$ as well as inconsistency in process ${ }^{14}$ because of the lack of national standards. ${ }^{15,16}$

Because UK applications for research governance approval are always arranged by local investigators rather than the coordinating centre, our data collection about the process of approval was retrospective. Despite the retrospective design of this study, the UK centres participating in the clinical trials were enrolled prospectively, so there were no omissions. However, by restricting inclusion in our survey to those trial centres where $R \& D$ approval had been obtained, we may have underestimated the impact of research governance by omitting lengthy delays at centres where R\&D approval had never been negotiated. Although we had to ascribe dates for seven dates of application $(12 \%)$ and three dates of approval (5\%), we thereby revealed the lack of an electronic or paper trail at some departments. Therefore, a prospective repetition of this study, with independent evaluation, would be worthwhile. The generalizability of our findings is affected by the survey 
being restricted to only four (albeit large) clinical trials at only one quarter of UK R\&D departments. Therefore, we cannot accurately analyse whether there are regional variations in the approval process. There were differences in the delays experienced by the four clinical trials in our study; these are likely to be due to the need for 'service support costs' negotiations in one trial (CLOTS), but less so in the others. This suggests that the UK needs a faster, streamlined approach to assessing the financial impact of clinical trials on Trusts. By examining the interval between application and final approval, we did not distinguish two important components of the R\&D approval process, which would be worthy of further scrutiny: the endorsement of governance arrangements versus hospital management approval.

This study has several implications for policymakers involved with 'busting bureaucracy' and those governing the research governance process. The lack of an electronic or paper trail at a few departments clearly necessitates a unified system - much like COREC's - for maintaining an audit trail. The online $\mathrm{R} \& \mathrm{D}$ form already presents a simple solution, but only half of R\&D departments currently use it. For studies that have few local issues, a multicentre process for research governance approval_again emulating COREC — would be welcome. ${ }^{14}$ Proposed multicentre solutions include the 'trial passport', already piloted in Manchester and promoted by the R\&D Forum and the UK Clinical Research Collaboration, and Multicentre Research and Development in Scotland. Last of all, reasonable timescales must be imposed on R\&D departments, much as COREC imposed them on research ethics committees. Whilst imposed timelines should be achievable within the current $\mathrm{R} \& \mathrm{D}$ infrastructure, they should not unduly delay the investigation of potentially beneficial or harmful treatments in clinical trials; a maximum delay of four weeks from application to approval would increase the number of patients recruited to clinical trials in the first couple of years by an eighth, and minimize wastage of scarce research funds.

Acknowledgments We are grateful to Gina Cranswick, Alison Clark, Karen Innes, Mary Paterson, Bridget Colam, and Lynn Horribine for providing details of the centres in the trials that they coordinate, and commenting on this manuscript.

Contributorship RAS had the idea for the study, analysed the data, and wrote the article. TMB collected the data. MSD, PAGS, PMW and CPW are the UK Chief Investigators of the trials in this study. Every author revised the manuscript critically for important intellectual content. All authors gave final approval of the version to be published. RAS is responsible for the overall content as guarantor.

Competing interests RAS, TMB, MSD, PAGS and CW have no competing interests. PMW has received reimbursement for expenses in attending international conferences from Siemens, Cordis, Boston Scientific, UK Medical, Microvention, he has been reimbursed by Pyramed UK for running an educational programme and holds a research grant from Microvention funding a randomized controlled trial (HELPS). PMW has received consulting fees from Boston Scientific, Cordis, UK Medical and Microvention.

Funding RAS is funded by the UK Medical Research Council. GALA is funded by the Health Foundation (UK), CLOTS and IST3 are funded by the UK Medical Research Council, and HELPS is funded by Microvention. The funding sources had no influence on the content of the manuscript.

\section{REFERENCES}

1 International Campaign to Revitalise Academic Medicine. Available at http://bmj.bmjjournals.com/academicmedicine/ (accessed 24/08/ 2006)

2 Warlow C. A new NHS research strategy. Lancet 2006;367:12-3

3 Department of Health. Best Research For Best Health: A new national health research strategy. London: HMSO, 2006

4 Pollock AM, McNally N, Kerrison S. Best research. BMJ 2006;332: 247-8

5 Al-Shahi R. Research ethics committees in the UK - the pressure is now on research and development departments. J R Soc Med 2005;98: 444-7

6 Kerrison S, McNally N, Pollock AM. United Kingdom research governance strategy. BMJ 2003;327:553-6

7 Sheard L, Tompkins CN, Wright NM, Adams CE. Non-commercial clinical trials of a medicinal product: can they survive the current process of research approvals in the UK? J Med Ethics 2006;32:430-4

8 Thornton H. Research governance: whose idea is it? BMJ 2006;332: 238

9 Slowther A, Boynton P, Shaw S. Research governance: ethical issues. $J$ $R$ Soc Med 2006;99:65-72

10 Shaw S, Boynton PM, Greenhalgh T. Research governance: where did it come from, what does it mean? J R Soc Med 2005;98:496-502

11 Department of Health. Research Governance Framework for Health and Social Care. 2nd edition. London: HMSO, 2005

12 Shaw S, Barrett G. Research governance: regulating risk and reducing harm? J R Soc Med 2006;99:14-9

13 Galbraith N, Hawley C, De-Souza V. Research governance: research governance approval is putting people off research. BMJ 2006;332:238

14 Dumville JC, Watson J, Raynor P, Torgerson DJ. Research Governance: a barrier to ethical research? QJM 2004;97:113-4

15 Elwyn G, Seagrove A, Thorne K, Cheung WY. Ethics and research governance in a multicentre study: add 150 days to your study protocol. BMJ 2005;330:847

16 Bond ME. Research governance is important. BMJ 2006;332:365 